\title{
ENTREVISTA
}

\section{LA INTERDISCIPLINARIEDAD COMO FORMA DE TRABAJO Y LOS MODELOS MATEMATICOS PARA COMPRENDER EL MUNDO}

\author{
A INTERDISCIPLINARIDADE COMO FORMA DE TRABALHO E OS \\ MODELOS MATEMÁTICOS PARA COMPREENDER O MUNDO
}

\section{THE INTERDISCIPLINARITY AS A FORM OF WORK AND MATHEMATICAL MODELS TO UNDERSTAND THE WORLD}

Stella Maris Vaira Facultad de Bioquímica y Ciencias Biológicas Universidad Nacional del Litoral

\section{RESUMEN}

En esta entrevista, las docentes-investigadoras Elena F. de Carrera y Liliana Nitti exponen desde sus experticias la importancia de la interdisciplinariedad, con profundo conocimiento en grupos de trabajo en el área de las Ciencias de la Salud, en el caso particular de Elena, que hizo que Probabilidad y Estadística fuese posible enseñarse a Médicos en ejercicio de su profesión. Liliana, en palabras simples, nos relata la historia del análisis armónico y su importancia en el contexto del desarrollo del hombre. Ambas enfatizan el valor de los Modelos Matemáticos como modo de comprender la realidad.

Palavras Claves: Interdisciplinariedad. Trabajo. Modelos Matemáticos.

\section{RESUMO}

Nesta entrevista, as docentes-pesquissadoras Elena F. de Carrera e Liliana Nitti expõem, a partir de suas perícias, a importância da interdisciplinaridade, com profundo conhecimento em grupos de trabalho na área das Ciências da Saúde, particularmente no no caso de Elena, que possibilitou o ensino de Probabilidade e Estatística a médicos em exercício de sua profissão. Liliana, em simples palavras, nos relata a história da análise harmônica e sua importância no contexto de desenvolvimento do homem. Ambas enfatizam o valor dos Modelos Matemáticos como modo de compreender a realidade.

Palavras-chave: Interdisciplinaridade. Trabalho. Modelos Matemáticos.

\begin{tabular}{|l|l|l|l|l|l|} 
Revista RBBA & ISSN 2316-1205 & Vitória da Conquista & V.9 n ${ }^{\circ} 1$ & p. 367-380 & Julho/2020
\end{tabular}




\title{
LA INTERDISCIPLINARIEDAD COMO FORMA DE TRABAJO Y LOS MODELOS MATEMATICOS PARA COMPRENDER EL MUNDO
}

\begin{abstract}
In this interview, the teaching-researchers Elena F. de Carrera and Liliana Nitti explain from their expertise the importance of interdisciplinarity, with deep knowledge in working groups in the area of Health Sciences, in the particular case of Elena, that made Probability and Statistics possible to teach Doctors in the exercise of their profession. Liliana, in simple words, tells us the history of harmonic analysis and its importance in the context of man's development. Both emphasize the value of Mathematical Models as a way of understanding reality.
\end{abstract}

Keywords: Interdisciplinarity. Work. Mathematical Models.

Nesta entrevista, as professoras-pesquisadores Elena F. de Carrera (https://orcid.org/0000-0002-9815-5531) e Liliana Nitti (https://orcid.org/0000-00029637-7704) explicam com seus conhecimentos a importância da interdisciplinaridade, com profundo conhecimento em grupos de trabalho na área de Ciências da Saúde, no caso particular de Elena, que tornou possível a Probabilidade e a Estatística ensinar os médicos no exercício de sua profissão. Liliana, em palavras simples, nos conta a história da análise harmônica e sua importância no contexto do desenvolvimento do homem. Ambos enfatizam o valor dos modelos matemáticos como uma maneira de entender a realidade.

Elena F. de Carrera es Profesora de Matemática del Instituto del Profesorado de la Universidad Nacional del Litoral (UNL). Licenciada en Matemática Aplicada (FIQUNL). Master Science Biometría, bajo la dirección de un Comité Consejero compuesto por el Dr. Hugo Aimar, el Dr. Martín Grondona y el Dr. Rodolfo Cantet de la Escuela de Graduados Facultad de Agronomía (UBA). Profesor Titular, Investigadora y Directora del Departamento de Matemática de la FBCB. Autora de más de 40 artículos de divulgación científica en Educación Matemática y ha dirigido más de 15 tesis de Maestría en el área, formó grupos de investigación, publicó libros y dirigió numerosos proyectos de investigación que fueron pioneros en su área desde la problemática de la metodología de resolución de problemas, el curriculum universitario, la deserción universitaria y la preocupación en la última década por la enseñanza de las ciencias y el trabajo interdisciplinario. Comenzó por el año 1994 a viajar y a dictar cursos de postgrado en el área de Probabilidad y Estadística, tanto en el país como en el exterior, sembrando la necesidad de fomentar el método científico para el análisis de datos. 


\section{LA INTERDISCIPLINARIEDAD COMO FORMA DE TRABAJO Y LOS MODELOS MATEMATICOS PARA COMPRENDER EL MUNDO}

Liliana Nitti. Profesora de Enseñanza Media en Matemática, Escuela Universitaria del Profesorado de la UNL. Licenciada en Matemática Aplicada (FIQ-UNL). Doctora en Matemática, su tema de Tesis Doctoral: Medidas que Duplican y Propiedades de Separación Métrica. Extensión de Medidas con la Propiedad de Duplicación, la realizó bajo la dirección del Dr. Hugo Aimar. Estudió matemática y se preocupó por la docencia tanto en el nivel medio como en el nivel universitario. Docente de la Facultad de Ingeniería Química, Facultad de Humanidades y Ciencias, Coordinadora de programas curriculares del Ministerio de Educación de la Provincia de Santa Fe, Investigadora y Docente.

"La educación no cambia el mundo, cambia a las personas que van a cambiar el mundo". Paulo Freire (1921-1997)

La interdisciplinariedad y la docencia universitaria se unen articulando y generando una nueva actitud frente al conocimiento, un proceso de aprender a ser profesor universitario constante. La interdisciplinariedad constituye una necesidad en el mundo actual dado el carácter complejo de la realidad que implica un abordaje multicompartimental no realizable desde disciplinas aisladas y con fragmentación del conocimiento. Si bien la Matemática, como disciplina, sigue creciendo, es cierto que muchos de los avances de la misma se debieron a la necesidad de resolver un problema real, y en cuyo caso quien trabajó en la resolución de ese problema, además le pareció tan interesante la matemática que se generaba, que terminó resolviendo "más de un problema". Para lograr la interdisciplinariedad desde el currículo es vital partir del trabajo docente, de todo el grupo de docentes que compone el espacio con el propósito de lograr objetivos generales relacionados con determinados conocimientos, habilidades y modos de actuación profesional que son clave en la formación de los estudiantes y que no es posible lograrlos con la debida profundidad desde la óptica de una sola disciplina o asignatura académica.

-Si bien todas las áreas de la matemática son importantes para la formación de un Profesor de Matemática, hay una en particular en la que Elena trabajó arduamente y formó a profesionales de las Ciencias Experimentales en general, Salud y Médicos en particular y es precisamente la Estadística. ¿Cómo vive esta importancia? y ¿cuál es el rol de esta disciplina en las Ciencias de la Salud?

Elena. Ya nadie duda que Probabilidad y Estadística son áreas de interés para todas las disciplinas y que se convirtieron por estos días en las herramientas fundamentales del método científico. Pero no solamente ellas, sino la Matemática en general: la modelización matemática 
en particular es aprovechada con frecuencia en distintas disciplinas, ya sea Modelo de Markov, Modelos Continuos, Modelos Discretos, para nombrar solo algunos, se aplican en las Ciencias Físicas, en la Astronomía, en las Ciencias Económicas, en la Bioquímica y en la Medicina.

Es evidente que la importancia de la Matemática no sólo se circunscribe al mundo actual, sino también en la antigüedad, donde encontramos que se marcaban los números con las cuñas óseas en la arcilla (allá por el año 3000 a.C.) con la escritura cuneiforme hasta hoy en que el mundo de las computadoras e informática hace que la necesidad Matemática sea cada vez mayor. No nos preocupamos, ni asustamos si Iannis Xenakis (Compositor e Ingeniero, 1922-2001) y la computadora nos dejaron la música estocástica y esta es sola una mención dentro del arte. Pero también está la Matemática en la Arquitectura: El Partenón y la Catedral de la Sagrada Familia del arquitecto catalán Gaudí en Barcelona, son vivo ejemplo de ello.

A la Matemática no sólo se la piensa como una aplicación; es una Ciencia que resulta transversal a muchas disciplinas, entre ellas la misma Medicina, la Genética, la Herencia, la Epidemiología que son algunas áreas de las ciencias de la salud; también hay Matemática "nueva" como la Geometría Fractal que hoy, por ejemplo, se la utiliza para el análisis de las bioimágenes de ciertos tipos de cáncer o en cualquier otra estructura compleja de la naturaleza. Volviendo al punto inicial, llegó en la década de los años '90 la necesidad de incorporar la Estadística como asignatura en todos los espacios curriculares de carreras universitarias, aún en la escuela media y primaria, independientemente del perfil u orientación de la misma. Esto obligó y me obligó a una adaptación de la disciplina para cada caso particular, dando origen a lo que se denomina Estadística Aplicada. Nos esperaban épocas de nuevos desafíos en el Departamento de Matemática de la Facultad de Bioquímica y Ciencias Biológicas de la Universidad Nacional del Litoral.

\section{-¿Qué papel tiene la interdisciplinariedad en la enseñanza y aprendizaje de la Matemática?}

Elena. La Matemática tiene sus bemoles, más bien una fama que a veces la presenta como "muy dura", este mito es lo que expande entre los alumnos y dificulta su aprendizaje. Es así que empecé a trabajar el tema de la interdisciplinariedad, interiorizándome primero para luego llevarlo a mi equipo de trabajo. En una carrera como Bioquímica no fue muy dificil formar equipos interdisciplinarios y lo volví a repetir en la carrera de Medicina. Nos pusimos a estudiar, 
a experimentar y le dimos a la Matemática un enfoque interdisciplinario, en realidad, transdisciplinario, es decir, fuimos más allá de la interdisciplinariedad porque seguíamos a Basarab Nicolescu que transgrede las fronteras entre las disciplinas. En logro fue facilitado por el haber cursado la Maestria en Biometría en la Ciudad de Buenos Aires: su currícula tiene un fuerte contenido estadístico con importantes enfoques hacias las Ciencias Biológicas, Químicas, Veterniaria, Agronómicas y Medicina.

La Matemática con este enfoque resulta más atractiva para los alumnos y nace en ellos el deseo de entenderla acercándose más a su objetivo de estudio y al docente le abre un panorama que lo enriquece. Se intenta comprender la complejidad del mundo en el que se está inmerso y como dice Edgar Morin el conocimiento es una aventura en espiral. Estoy convencida de que el trabajo interdisciplinario con las otras materias del currículo hace visible la potencialidad de la matemática. Esta acción permite marcar su fuerte relación con otras disciplinas y su poder de interpretar la realidad en la que nos encontramos. Esto es fundamental no sólo para el estudiante en el nivel superior sino para un simple ciudadano, porque se necesitan resolver, o al menos reconocer, los problemas de origen matemático que se presentan en la vida en sociedad.

\section{-¿Cómo forma los grupos interdisciplinarios?}

Elena. Desde que inicié mi trabajo en la docencia universitaria, pude conformar grupos de trabajos, y siempre fueron interdisciplinarios. Al principio estudiábamos matemática para dar clases a los futuros matemáticos, allí era necesario hacer conexiones internas de la propia Matemática. Luego fuimos buscando nuevos horizontes profesionales y empecé a enseñar matemática para no matemáticos, y allí el desafío fue mayor. El grupo de docentes con el que trabajé (con algunos por más de 30 años) no sólo estudió para perfeccionarse, sino que entendió la importancia de redoblar el trabajo con la mira centrada en la comunidad. Tuvimos el apoyo de los científicos, de las autoridades de la Universidad, de la propia Facultad y trabajamos en forma conjunta con las cátedras de Química, Biología y Física (del ciclo básico) y muchas otras del ciclo superior. Así surgió un material para un curso de ingreso en la Facultad en el año 1989 llamado CARDI (Curso de Articulación Disciplinar a Distancia). Pero este trabajo mancomunado hizo surgir la necesidad de integrar los saberes de esas materias, las formas de enseñar y de comenzar compenetrándonos en la interdisciplinariedad, como consecuencia de Revista RBBA $\mid$ Revista Binacional Brasil Argentina 
todo esto se generaron varios líneas de investigación, más de 14 proyectos y programas, tendientes a ser empáticos con la problemática del estudiante (inicialmente de Bioquímica) que estudiaba carreras donde la Matemática no era el eje central de ellas; no obstante, siempre entendí y entendimos con mi equipo, la importancia que la disciplina encerraba. La matemática es una disciplina, como dice Ian Stewart, que trabaja en "bambalinas".

\section{-Elena trabajó un tema muy interesante sobre Modelos Matemáticos para Tuberculosis, ¿qué la motivó?}

Motivó el tema de tuberculosis el hecho de que la enfermedad a la que se daba por controlada, había empezado a recrudecer en todo el mundo según datos de la OMS (Organización Mundial de la Salud, allá por los años 1997-1998. Me interesó y me interesan los Modelos Matemáticos, para el caso de un proceso epidemiológico de una enfermedad es necesario conocer los compartimentos, la población se divide en compartimentos, cada individuo pertenece a uno de ellos. Cuando comencé a estudiar el tema, los modelos matemáticos de la enfermedad eran determinísticos, y más realista, aunque tal vez más difícil de analizar, es considerarlo como estocástico.

\section{- ¿Nos recuerda un poco más del tema y de qué se trató?}

En la Universidad de Buenos Aires estudié la carrera de Maestría en Biometría que cursé y terminé con la elección de un tema muy relacionado con la Biología, la Epidemiología y la Salud Pública y también con la Matemática, la cual fue precisamente la tesis, se llama "Un modelo estocástico de los estados de la transmisión de la tuberculosis".

Se han introducido modelos estadísticos y matemáticos para entender mejor la dinámica de esta enfermedad, caracterizada por un período de latencia indefinido y una curación que no crea inmunidad. Junto a mis directores del trabajo de Tesis, Dr. Martín Grondona y el Dr. Hugo Aimar, nos concentramos en 3 (tres) objetivos, a saber: i) Hallar un modelo estocástico de la tuberculosis contemplando tres compartimientos que se correspondan con estadios de la enfermedad y las características de la misma; ii) obtener expresiones para los momentos de orden uno y dos, con el objetivo de estimar el número esperado de individuos en esos compartimientos; iii) analizar el modelo propuesto a través de simulaciones, ya que en ese 


\section{LA INTERDISCIPLINARIEDAD COMO FORMA DE TRABAJO Y LOS MODELOS}

MATEMATICOS PARA COMPRENDER EL MUNDO

momento no pudimos acceder a una base de datos completa para estimar los parámetros del Modelo. El trabajo fue de naturaleza teórica (no experimental). Para modelar la enfermedad, tratando de cuantificar sus particularidades, se estudiaron varios modelos estocásticos, hasta llegar a describir un modelo estocástico compartimental. Con respecto a los compartimentos se consideraron tres: individuos susceptibles, individuos latentes e individuos infecciosos. A diferencia de los modelos más difundidos en epidemiología matemática (SIS, SIR y SIRS), el desarrollado en este trabajo incluye una clase latente y contempla, además, la recuperación de los infecciosos por efecto del tratamiento y su reingreso a la clase latente. La trascendencia del modelo se basa en que está formulado como una cadena de Markov a tiempo continuo, variable que tiene un rol fundamental, especialmente en la duración indeterminada de la latencia. El Modelo es un sistema de ecuaciones diferenciales en probabilidad condicional, que caracteriza la transición entre los distintos estados del proceso, y es una modificación del sistema estocástico básico de Kolmogorov (1931). Dada la complejidad de su resolución, se realizaron ciertas suposiciones sobre las distribuciones a las que pueden ajustarse los parámetros del modelo y por el método de la función generatriz se obtuvieron los momentos de primer y segundo orden (los que dan origen a la media y variancia de una variable aleatoria). Se deduce que el proceso estocástico modelado es una forma especial del análisis de Fourier. El modelo estocástico propuesto demostró funcionar correctamente con los datos de la simulación: permitió detectar la predisposición de algunos individuos a desarrollar la enfermedad, hecho que coincide con la observación clínica. El modelo desarrollado en este trabajo permitió cuantificar la incertidumbre generada por la dinámica entre los distintos estadíos de la tuberculosis. Fue una experiencia de trabajo muy rica, y aún se habla y se desarrollan temas (incluso Tesis Doctorales) en Modelos Matemáticos para distintas enfermedades. Creo que después del Premio Nobel en 1902 otorgado a Ronald Ross (nació en la India, médico, matemático, entomólogo), quien demostró que eliminando los mosquitos se eliminaría la malaria, se estableció en la cronología de los modelos un antes y un después en estos temas. Y en estos días con esta pandemia de coronavirus que nos acecha como sociedad, la modelización matemática es imprescindible ya sea determinística o estocástica. 
-Durante mucho tiempo no se reflexionó sobre el tema de género en la docencia universitaria, no recuerdo (pensando en la década del 90, a fines del siglo pasado), que hubiera debates en ese campo en esa época. Estas dos docentes-investigadoras, Elena y Liliana, siguieron mandatos internos, gusto por la Matemática y por hacer lo que a ellas les gustaba, sin pensar si era un espacio para sus compañeros varones o si las mujeres también podían ser parte de los grupos de "exactos". ¿Qué les llevó a iniciar los estudios de matemáticas?

Liliana. Bueno, mis estudios hacia la matemática podría decirse comenzaron, por cuestiones de disidencia familiar o mejor dicho por cuestiones de género. Todo se remonta a la época cuando terminé la escuela primaria y debía elegir en donde cursaría la escuela secundaria. Siempre había sido una alumna muy inquieta y según las buenas lenguas bastante inteligente. Aprendía con facilidad, sobre todo lo relacionado a las ciencias exactas y naturales. Mi ilusión era estudiar una vez terminada la escuela primaria, en la Escuela Industrial Superior, que depende de la Universidad Nacional del Litoral, institución de gran prestigio para la época y aún en la actualidad. Consciente de lo que significaba ingresar a la misma, a la cual se accedía por un durísimo examen de ingreso, comencé a prepararme para acceder a la carrera de Técnico Químico, para luego seguir la carrera de Ingeniería Química. Ese era mi plan...

Cuando, inocentemente hice conocer mi decisión a mis padres, la negativa fue absoluta. Los argumentos de tal reacción fueron, palabras textuales:- ¡No van mujeres a dicha escuela, es sólo para hombres!- Era cierto, en la Escuela Industrial Superior, sobre aproximadamente 500 alumnos, (por dar una estimación) en los comienzos de los años 60 eran menos de 10 mujeres quizás las que asistían. Jamás había considerado dicha variable en mi cabeza. La fuerza de la familia fue contundente, imaginem padres italianos, con dos hijos que eran la primera generación en la posguerra en Argentina. Había un mandato implícito, estudiar y no arriesgarse al fracaso ni a la deshonra. Todo esto y otros argumentos hicieron que yo dócilmente a mis doce años, me inscribiera en otra muy buena escuela: la famosa escuela Normal Superior (allí la relación hombre/mujer era exactamente a la inversa del otro colegio). Todos quedaron contentos y yo... con un futuro incierto. Pero bueno, gracias a las buenas ondas del universo... todo se desarrolló con normalidad. Me fue muy bien, y lo mejor fue que conocí a excelentes profesores, pero lo más importante capté algo propio e innato del tipo de escuela: el interés por el cómo enseñar y contener a los alumnos. Esta cuestión me hizo olvidar de alguna forma mi historia anterior y cuando terminé el secundario me decidí por estudiar Profesorado de Matemática, carrera que se dictaba en ese momento en la Escuela Universitaria del Profesorado Revista RBBA Revista Binacional Brasil Argentina 


\section{LA INTERDISCIPLINARIEDAD COMO FORMA DE TRABAJO Y LOS MODELOS MATEMATICOS PARA COMPRENDER EL MUNDO}

que dependía de la Universidad Nacional del Litoral (que con los años se transformaría en la Facultad de Humanidades y Ciencias). Me sentía muy cómoda, tuve excelentes profesores y mejores compañeras... (ah...y lo importante es que prevalecía el número de mujeres.... por sobre el de los hombres y esto mantuvo a mis padres súper tranquilos!!!).

En el país... comenzaban épocas políticas muy convulsionadas y el tipo de amistad que yo podría tener en dicho ámbito, les daba cierta tranquilidad pues no corría el riesgo según sus pensamientos, de conectarme con grupos de estudiantes activistas. ¡La niña estaba a salvo! bueno, para la cabeza de ellos esto era así.

En el Profesorado de Matemática, no sólo canalicé mi interés por la ciencia, sino también el aprender a conjugarla con la enseñanza, cuestión que marcó mi accionar futuro. Prontamente empecé a trabajar en la misma institución y en la Facultad de Ingeniería Química como ayudante de cátedra, pero era consciente que para poder hacer docencia en el nivel universitario era necesario profundizar el saber matemático y por sobre todo debía hacer investigación. Es así que cursé la Licenciatura en Matemática y luego el Doctorado en Matemática, versando mi tesis en sobre teoría geométrica de la medida.

Trabajé en investigación matemática, en el instituto de investigaciones de matemática aplicada del litoral, IMAL, de doble dependencia Conicet-UNL, y tuve la suerte de conocer y trabajar junto a prestigiosos investigadores e investigadoras en temas que pude profundizar relacionados a los de mi tesis.

En el ámbito de la investigación la sensación es maravillosa uno siente que comienza a explorar y que está ante un universo que se agranda cada vez más, aparecen cosas nuevas, inéditas pero también desde algún punto de vista surge lo ya conocido, disfrazado bajo otra óptica, bajo otra dimensión, aparece la creación...

Todas estas sensaciones, traté de trasmitirlas en mis clases, lo que me permitió estar también interesada en la esencia del porqué y cómo enseñar y propiciada por el hecho de trabajar en una Facultad de Humanidades y dictar mis cátedras en el Profesorado de Matemática. Tuve la oportunidad de realizar Investigaciones sobre enseñanza de la matemática en cuestiones que intenté plasmar en algunos trabajos y en tesis que he dirigido. Hoy en día, puedo decir, que esta doble visión, investigación matemática-enseñanza, fue muy enriquecedora para mi quehacer y para quienes tuve de alumnos a lo largo de casi 40 años de docencia. Moraleja...¿mis padres tenían razón o sólo fue una casualidad que me brindó la vida? 
-Todos los que hemos pasado por un curso regular de Matemática en la Universidad, desde los que estudian en los Profesorados o Licenciaturas en Matemática, hasta los estudiantes de Bioquímica, profundizan el tema de funciones y las "deformaciones" de las funciones trigonométricas: $y=\operatorname{sen}(2 \pi n x), y=\cos (2 \pi n x)$ con $n \in \mathbb{Z}$ en ellas hay riqueza y complejidad de conceptos conocidos, son bellas de estudiar y caen en la familia de las funciones que podemos derivar e integrar sin problemas, representar gráficamente a algunas de ellas o incluso una combinación lineal reproduce muchos de los movimientos ondulatorios conocidos. Una de las cuestiones que inicialmente intrigó más a los matemáticos, a partir de las ideas de Fourier: ¿cómo es que una serie de términos, muy "suaves" como son las funciones "senos", "cosenos" y combinaciones lineales de ellas, sea capaz de representar una función con una discontinuidad en un punto? Por ellos surge la pregunta a la Doctora Liliana Nitti: ¿qué es el Análisis Armónico y qué aportes tiene al desarrollo de un pueblo?

Para responder sobre qué es el Análisis Armónico, permítanme hacer un breve recorrido histórico sobre la historia de la matemática, tratando de no usar símbolos y escritura matemática muy específicos.

El hombre desde el comienzo de su existencia se preguntó sobre cuestiones de la naturaleza y por todo lo que ella representa. Los fenómenos recurrentes como las estaciones, las mareas, las fases de la luna, la composición del sonido, entre otros, fueron los hechos sobre los cuales los antiguos filósofos necesitaban responder para comprender el comportamiento del universo.

El indagar sobre la composición de los procesos recursivos es lo que guió a los matemáticos, sobre todo a los de la antigua Grecia, a encontrar a algún princípio que rigiera el Cosmos. Pitágoras (siglo VI a. C.) hallaría una clave que se consolidaria a través de los siglos: el número.

El motivo fundamental por el cual los pitagóricos mantenían a los números como principio de todo, fue que notaron que muchos fenómenos observables son traducibles en términos matemáticos: consideraban que el universo es un todo armónico, cuantitativamente medible. Medible matemáticamente son los sonidos, las notas musicales, los ciclos de los astros, de las estaciones, de las mareas y otros acontecimientos. Bajo esta visión los números son los ordenadores del universo.

Pero uno de los logros de Pitágoras sobre la naturaleza aritmética del cosmos fue mostrar la armonía que producían los sonidos de los instrumentos de cuerda. Lo que Pitágoras descubrió es que al dividir la cuerda en ciertas proporciones ésta era capaz de producir sonidos distintos 


\section{LA INTERDISCIPLINARIEDAD COMO FORMA DE TRABAJO Y LOS MODELOS}

MATEMATICOS PARA COMPRENDER EL MUNDO

y agradables para el oído. Era una maravillosa confirmación de su teoría. Pitágoras dividió la cuerda en doce partes y buscó los intervalos que producían un sonido agradable y se dio cuenta que, si establecía determinadas longitudes, proporcionales a 12, los sonidos que se producían eran placenteros. Números y belleza musical conformaban así una sola entidad. Establece, con ello, una serie de fundamentos donde la tensión de la cuerda, la propagación y frecuencia de un sonido estaban relacionadas.

Muchos siglos después la inquietud pitagórica en cuantificar el sonido aparece en la modernidad con el análisis armónico y de alguna forma se cierra ciclo histórico - matemático y sienta las bases para proyecciones e investigaciones futuras.

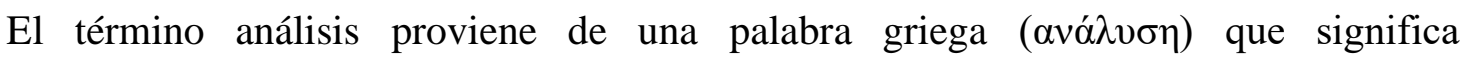
descomposición; esta sugiere separar o distinguir las partes componentes de algo (como una sustancia, un proceso, una situación) para descubrir su verdadera esencia. En concordancia el análisis armónico se ocupa, a grandes rasgos, de la descomposición de funciones en tonos puros que se llaman armónicos. Muy ligeramente, consideramos tonos puros a ciertos objetos que nos recuerdan a las funciones $\operatorname{sen}(2 \pi n x), \cos (2 \pi n x) \operatorname{con} n \in \mathbb{Z}$, las cuales aparecen en los desarrollos de Fourier.

Estos desarrollos no son más que una descomposición de la función original en una suma infinita de funciones elementales en senos y cosenos que tienen frecuencias múltiplos de la "señal" inicial que converge puntualmente a una función periódica y continua a trozos (o por partes).

El matemático inglés Brook Taylor (1685-1731) plantea en su obra de 1715, "Método de los Incrementos", el problema de describir el movimiento de una cuerda tensa (como por ejemplo puede ser la de un violín) y de establecer el tiempo de vibración sabiendo la longitud y el peso de la cuerda, y la fuerza que la tensa.

Los fundamentos matemáticos en los que se basa la solución de este problema, son dados casi cien años después por el matemático francés Jean Baptiste Joseph Fourier (17681830) en su obra llamada “Teoría Analítica del Calor” que publicó en 1822. Desde el punto de vista matemático, se obtiene una función discontinua a partir de la combinación de funciones continuas. Esta fue la atrevida tesis defendida por Fourier ante la Academia Francesa, que motivó severas objeciones de los matemáticos más importantes de su época como Lagrange y Laplace.

Revista RBBA $\mid$ Revista Binacional Brasil Argentina 


\section{LA INTERDISCIPLINARIEDAD COMO FORMA DE TRABAJO Y LOS MODELOS}

MATEMATICOS PARA COMPRENDER EL MUNDO

La coyuntura histórica provocada por la revolución industrial propiciada por la invención de la máquina a vapor a fines del siglo XVIII, explica por qué el investigar sobre la difusión del calor se vio como un problema neurálgico que trascendería a la época y fue de una influencia decisiva en el desarrollo de las matemáticas. En su libro "Teoría Analítica del Calor" planteó las bases de un teorema que después sería una herramienta de gran valor para las ciencias: "Toda onda compleja periódica se puede representar como la suma de ondas simples".

Dicho de otra forma, lo que establece este teorema, es que cualquier señal periódica (de la forma que sea) puede descomponerse como la suma de señales sinusoidales de distintas frecuencias. Nuevamente la recurrencia es fundamental... para estas interpretaciones.

Daniel Bernoulli había estudiado series de este tipo en la resolución del problema de la cuerda vibrante y Euler (1707-1783) había calculado los coeficientes de senkx y coskx en el desarrollo mencionado, pero a partir de Fourier con la posibilidad de expresar una función mediante una serie trigonométrica empezó a ser ampliamente discutida.

El análisis de Fourier juega un papel central en el análisis armónico, sobre todo en el tratamiento de señales; su contexto inicial para el estudio de la disipación del calor en un medio sólido, pronto lo llevaron al desarrollo de soluciones para la ecuación de Laplace y la ecuación de Onda. Algunas señales muestran componentes periódicos que se repiten a intervalos fijos; estos son estudiados convenientemente por el análisis basado en las series de Fourier. Algunas señales muestran componentes periódicos que se repiten a intervalos fijos; estos son estudiados convenientemente por el análisis basado en las series de Fourier.

Resulta que (casi) cualquier tipo de onda se puede escribir como una suma de senos y cosenos. Entonces, por ejemplo, si tuviera que grabar mi voz por un segundo diciendo algo, su serie de Fourier puede verse así: $\operatorname{voz}=\sin (\mathrm{x})+1 / 10 \sin (2 \mathrm{x})+1 / 100 \sin (3 \mathrm{x})+\ldots$

Las series de Fourier nos permiten obtener el espectro de frecuencia de señales continuas y periódicas. Pero, desafortunadamente, este tipo de señales no son tan frecuentes en la práctica como las no-periódicas. Esta situación requiere el desarrollo de una teoría matemática más ambiciosa y para ello aparece la Transformada de Fourier que solo simplificando mucho, se puede decir que, La transformada de Fourier es una operación matemática que rompe una señal en sus frecuencias constituyentes.

Este recorrido histórico, que comenzó con los experimentos realizados por los pitagóricos con un monocordio, documentado por Ptolomeo en su obra Harmónicos hacia el Revista RBBA Revista Binacional Brasil Argentina 


\section{LA INTERDISCIPLINARIEDAD COMO FORMA DE TRABAJO Y LOS MODELOS MATEMATICOS PARA COMPRENDER EL MUNDO}

año 150 d. C., y que llega hasta la modernidad con el problema de la cuerda vibrante tienen una gran conexión. De hecho, puede considerarse que el problema de los sonidos en el monocordio es un problema simplificado del que luego se abordaría en el de la cuerda vibrante.

La cuerda de un violín, o cualquiera de nuestras cuerdas vocales, crea un sonido que en la mayoría de los casos podemos oír. Las ondas son perturbaciones que se propagan en el espacio transportando energía y... están en todas partes... Por ejemplo, nuestra vista, en general, detecta ondas de radiación electromagnética, y este tipo de ondas, que no necesitan un medio material para propagarse, son la base de la radio, la televisión, el radar, los teléfonos celulares, y todo lo que compete a señales y comunicaciones. También son ondas las producidas por un terremoto, llamadas ondas sísmicas. Entonces, el descubrir qué leyes gobiernan las ondas nos puede ayudar a predecir un tsunami o a mejorar la comunicación satelital. Todo esto se desarrolla bajo muchas teorías del análisis armónico. Es por eso que a lo largo de los siglos XIX, XX y en el siglo actual el análisis armónico se ha convertido en un área enorme con innumerables aplicaciones en campos diversos como el procesamiento de señales, geofísica, eléctrica, tomografías, la mecánica cuántica o la neurociencia.

Claramente, para el desarrollo de un pueblo o una sociedad competente, es fundamental tener estudiosos e investigadores en esta área o en áreas relacionadas, pues la misma favorece al crecimiento en numerosos campos de la ciencia y la técnica. En nuestro país existen equipos de matemáticos relevantes que trabajan en temas muy actuales con respecto al análisis armónico; que se interconectan con matemáticos de todo el mundo y han obtenido logros en muchas áreas de las mencionadas anteriormente. 


\section{SOBRE LA ENTREVISTADORA}

Stella Maris Vaira es Licenciada en Matemática Aplicada egresada de la Facultad de Ingeniería Química (FIQ) de la Universidad Nacional del Litoral (UNL), Santa Fe, Argentina. Especialista en Estadística Aplicada (INNIE, Instituto Nacional de Investigaciones en Estadística de la Universidad Nacional de Tucumán). Magister en Matemática Aplicada (IMAL, Instituto de Matemática Aplicada del Litoral-CONICET y FIQ - UNL). Cursa el Doctorado en Estadística de la Facultad de Ciencias Económicas y Estadística de la Universidad Nacional de Rosario. Profesora Asociada, dedicación exclusiva "A" regular u ordinario en la FBCB-UNL.

Dirección electrónica:

Orcid: https://orcid.org/0000-0002-4857-7509

Recebido em: 19/05/2020

Aprovado em: 08/06/2020

Publicado em: 01/07/2020 